\title{
PERIODISMO Y AGENDA SETTING: UNA DISCUSIÓN SOBRE EL INTERÉS POR ASUNTOS PÚBLICOS DE LA CIUDADANÍA
}

\author{
JOURNALISM AND AGENDA SETTING: A DISCUSSION ON THE \\ CITIZENS' INTEREST IN PUBLIC AFFAIRS
}

\author{
Carolina Carazo Barrantes*
}

\begin{abstract}
RESUMEN
En este artículo se reflexiona sobre la capacidad de los medios para fijar la agenda de discusión pública de la ciudadanía en el contexto actual. Se discute la teoría de la agenda setting $y$ su evolución temática $y$ temporal en los últimos cuarenta años, asimismo se reflexiona en torno a tres asuntos específicos: 1) la problematización de lo que significa un clic, 2) la necesidad de orientación como generadora de interés por asuntos públicos y 3) el interés de la ciudadanía y su manera de participar en política en la actualidad. Se presentan ejemplos de dos campañas presidenciales (2014 y 2018) en Costa Rica. A pesar de la preferencia del público por el consumo de noticias sobre asuntos que no son de interés público, también se identifican pistas que apuntan hacia una ciudadanía con, por lo menos, algún grado de interés sobre asuntos públicos.
\end{abstract}

PALABRAS CLAVE: MEDIOS DE COMUNICACIÓN * PARTICIPACIÓN POLÍTICA * REDES SOCIALES * INTERÉS EN ASUNTOS PÚBLICOS * CIUDADANÍAS

\section{ABSTRACT}

This article reflects on the ability of the media to set the citizens' agenda for public discussion in the current context. It discusses the agenda setting theory and its thematic and temporal evolution over the last forty years and reflects on three specific issues: 1) the problematization of what a click means, 2) the need for orientation as an interest generator for public affairs and 3) citizens interest and their way of participating in politics today. The article presents examples of two presidential campaigns (2014 and 2018) in Costa Rica. In spite of the public's preference for news on non-public affairs issues, the article also identifies clues that point to a citizenship with, at least, some degree of interest in public affairs.

KEYWORDS: MASS MEDIA * POLITICAL PARTICIPATION * SOCIAL MEDIA * INTEREST IN PUBLIC AFFAIRS * CITIZENSHIP

Escuela de Ciencias de la Comunicación Colectiva (ECCC) y Centro de Investigación en Comunicación (CICOM), Universidad de Costa Rica.

carolina.carazo@ucr.ac.cr 


\section{INTRODUCCIÓN}

El periodismo es un pilar de la vida en democracia. En los últimos años, los cambios que ha sufrido la industria han sido vertiginosos y cabe preguntarse de qué manera y en qué medida estos cambios han tenido impacto en la esfera pública y la vida democrática.

Según el reconocido centro de investigación estadounidense Pew (Research Center), hace veinte años, solamente el $12 \%$ de los adultos estadounidenses consumía noticias en Internet. Hoy, ese número se sitúa en el $81 \%$. Cerca de seis de cada diez (62\%) accede a las noticias a través de las redes sociales - una cifra que se eleva al 84\% para las personas de 18 a 29 años de edad-. A medida que los consumidores han cambiado la forma en que acceden a las noticias, también tienen nuevas formas de interactuar con estas (Mitchell, 2016).

Pero los cambios van más allá y son mucho más profundos. En palabras de Bell (2016), directora del Centro Tow de Periodismo Digital de la Escuela de Periodismo de la Universidad de Columbia en Nueva York:

Las redes sociales no sólo se han tragado el periodismo, se han tragado todo. Se han tragado las campañas políticas, los sistemas bancarios, las historias personales, la industria del ocio, las ventas al por menor, incluso el gobierno y la seguridad. El teléfono en el bolsillo es nuestro portal al mundo. Creo que, en muchos sentidos, esto anuncia oportunidades enormemente excitantes para la educación, la información y la conexión, pero también trae consigo una serie de contingencias (parra. $3^{1}$, traducción propia ${ }^{2}$ ).

$1 \quad$ Social media hasn't just swallowed journalism, it has swallowed everything. It has swallowed political campaigns, banking systems, personal histories, the leisure industry, retail, even government and security. The phone in our pocket is our portal to the world. I think in many ways this heralds enormously exciting opportunities for education, information, and connection, but it brings with it a host of contingent existential risks.

2 Todas las citas en inglés fueron traducidas por la autora. Los textos originales están en citas en cada página.
En efecto, las tecnologías de información y comunicación han traído profundos cambios a la manera en que los ciudadanos buscan información, la consumen y la comparten. Las redes sociales y los algoritmos que las rigen han transformado la manera como los ciudadanos acceden a la información y también han permitido potenciar las posibilidades reales de incidencia que la gente común tiene sobre la función pública y el acontecer nacional.

El presente artículo se enmarca en la tradición de los estudios de fijación de la agenda (agenda setting), que se preguntan ¿quién fija la agenda de discusión pública? Como se verá, la respuesta hasta hace poco había apuntado, contundentemente, hacia los medios de comunicación tradicionales; sin embargo, el vertiginoso crecimiento de las redes sociales y las cada vez más complejas dinámicas entre las agendas de los medios, de los políticos y de la ciudadanía, entre otras, hacen que sea imperativo hacerse la pregunta nuevamente $y$ reflexionar no solo sobre la agenda de los medios sino también sobre la ciudadanía: ¿qué tipo de contenido le interesa?, ¿consume noticias sobre asuntos públicos?, ¿las utiliza para debatir y participar en la esfera pública?

Para responder a estas preguntas, este artículo se enfoca en la capacidad de los medios para fijar la agenda de discusión pública de la ciudadanía en el contexto actual. Se inicia entonces con una discusión de la teoría de la agenda setting y su evolución temática $y$ temporal en los últimos cuarenta años. Posteriormente, se considera la pertinencia de la teoría para responder a algunos de los asuntos cruciales que caracterizan la ecología mediática contemporánea. Se reflexiona en torno a tres asuntos específicos: 1) la problematización de lo que significa un clic, 2) la necesidad de orientación como generadora de interés por asuntos públicos y 3) el interés de la ciudadanía y su manera de participar en política en la actualidad. Mediante esta discusión, también se busca complementar y poner en perspectiva los demás artículos sobre el tema que integran este volumen. 


\section{AGENDA SETTING: LA EVOLUCIÓN DE LA TEORÍA}

La teoría de la fijación de la agenda nació hace más de cuarenta años cuando en 1972, McCombs y Shaw publican el primer estudio sobre el tema. Con su investigación sobre el poder de los medios de comunicación para fijar la agenda del público durante una campaña electoral en la comunidad de Chapel Hill, se dio inicio a una corriente de investigación que ha producido cientos ${ }^{3}$ de estudios empíricos en los más diversos contextos sociales.

McCombs (2014) asegura que fue la "elocuentemente argumentada tesis" (p.394) de Walter Lippmann sobre los medios como fuente primaria de "las imágenes en nuestras cabezas" (p. $39^{5}$ ) la que dio pie a la teoría de la agenda setting. Para McCombs, se trata de una teoría de las ciencias sociales que mapea con bastante detalle la contribución que hacen los medios de comunicación a lo que el público sabe sobre política y asuntos públicos: la idea teórica básica es que los elementos prominentes en la agenda de los medios no solo se hacen prominentes en la agenda del público, sino que también son considerados por el público como especialmente importantes.

En cuarenta años de historia, la teoría ha evolucionado. El propio McCombs (2014) habla de tres niveles: en el primer nivel, las investigaciones responden a la pregunta "¿sobre qué pensamos?" (sobre qué asuntos o issues en inglés); el segundo nivel se pregunta "¿cómo pensamos sobre esos asuntos?, ¿cuáles son sus atributos?"; $y$ el tercer nivel, que es relativamente nuevo (como se verá más adelante, sus inicios datan del año 2011) investiga sobre el poder de los medios para transferir las relaciones que hay entre asuntos y atributos a la agenda del público.

La teoría, que ha sido comprobada en numerosos estudios (ver un reciente repaso en McCombs, 2005 y 2014; McCombs y Reynolds,

3 David Weaver, en Johnson (2014, p. xi) afirma que la más reciente estimación asciende a 500 artículos publicados sobre agenda setting, sin contar cientos de ponencias y numerosos libros y capítulos de libros.

4 Eloquently argued thesis.

$5 \quad$ The pictures in our heads.
2009 y McCombs, Shaw y Weaver, 2014), establece que los medios tienen el poder de fijar la agenda del público; es decir, se ha demostrado que el público sí considera los asuntos o temas que los medios proponen como importantes $y$ por lo tanto, la respuesta a la pregunta "¿sobre qué temas pensamos?", es sobre los asuntos a los que medios de comunicación les dan importancia. La investigación sobre la fijación de la agenda avanzó a su segundo nivel centrándose en los atributos de los asuntos de la agenda de los medios y la del público. McCombs (2014) explica por qué este era el siguiente paso natural en el desarrollo de la teoría:

Los asuntos públicos, como todos los demás objetos, tienen atributos. Algunos aspectos de los asuntos, es decir, algunos atributos, se destacan en las noticias y en la manera en que la gente piensa y habla sobre los asuntos. Además, los atributos más destacados de un determinado asunto a menudo cambian con el tiempo. [...] esto es especialmente cierto para la economía, un tema importante para muchos países en las últimas décadas. A veces el atributo prominente de la economía es la inflación; en otros momentos es el desempleo o los déficits presupuestarios. La agenda setting de atributos amplía nuestra comprensión de cómo los medios de comunicación moldean la opinión pública sobre los temas del día (p. $49^{6}$ ).

Teóricamente, mientras que la teoría del establecimiento de la agenda de primer nivel sugiere que los medios de comunicación

$6 \quad$ Public issues, like all other objects, have attributes. Some aspects of issues, which is to say, some attributes, are emphasized in the news and in how people think and talk about issues. Moreover, the salient attributes of a particular issue often change over time. [...] this is especially true for the economy, a major issue for many countries in recent decades. Sometimes the prominent attribute of the economy is inflation; at other times it is unemployment or budget deficits. Attribute agenda setting extends our understanding of how the news media shape public opinion on the issues of the day. 
pueden afectar lo que pensamos, el segundo nivel sostiene que los medios también pueden influir en cómo pensamos sobre los objetos (Carroll and McCombs, 2003; Kiousis et ál., 2006; McCombs and Reynolds, 2009; Wang y Shoemaker, 2011; Wanta, Golan y Lee, 2004 citados por Kiousis et ál., 2015, p. 366).

En el año 2011, Lei Guo y Maxwell McCombs presentan dos ponencias ${ }^{7}$ en las que se habla por primera vez del tercer nivel de la teoría de agenda setting: la premisa principal del tercer nivel es que los medios de comunicación no solo dicen en qué pensar y cómo pensar en ello, sino que "también son capaces de decirnos qué asociar y cómo hacerlo" (Vu, Guo y McCombs, 2014, p. 6698).

La evolución al tercer nivel se da por la exploración de nuevas posibilidades metodológicas que incluyen el análisis de redes sociales (social network analysis, en inglés). De hecho, Guo (2012) publica un artículo sobre lo que llama "una exploración metodológica" de la aplicación del social network analysis en la corriente de investigación de agenda setting y ya en sus ponencias del año 2011, McCombs y Guo acuñaron el término "Network Agenda Setting Model" (nas).

Para Vu, Guo y McCombs (2014), la propuesta del NAS significó un gran cambio en la forma en que se piensa acerca de los efectos mediáticos: la investigación tradicional siempre ha asumido que los asuntos (issues) se trasladan como elementos individuales de la agenda de los medios a la agenda del público. El nuevo modelo nas teoriza que los asuntos y sus atributos pueden transferirse simultáneamente en "paquetes" (bundles) entre una agenda y la otra.

$7 \quad$ Lei Guo and Maxwell McCombs, "Network Agenda Setting: A Third Level of Media Effects" (paper presented at the annual conference of the International Communication Association, Boston, MA, May 2011); Lei Guo and Maxwell McCombs, "Toward the Third-Level AgendaSetting Theory: A Network Agenda-Setting Model" (paper presented at the annual conference of the AEJMC, St. Louis, MO, 2011) en Vu, H. T., Guo, L., \& McCombs, M. E., 2014, p. 683.

They are also capable of telling us what and how to associate.
La corriente de estudios de agenda setting de tercer nivel es muy reciente $y$ son pocos todavía los trabajos que han explorado esta nueva avenida de investigación (Guo, 2012; Vu, Guo y McCombs, 2014; Vargo et ál., 2014; Kiousis et ál., 2014; Guo y Vargo, 2015). Para Guo y Vargo (2015) "el modelo nas contribuye a la discusión de las nuevas teorías de los medios y de la comunicación, permitiendo una representación más matizada de las redes de información $y$ ofreciendo un abordaje para medir de mejor manera el flujo de dichas redes entre diferentes entidades" (p. 558 ${ }^{9}$ ).

\section{AGENDA SETTING EN EL NUEVO CONTEXTO}

En un contexto mediático hipersaturado, con nuevas plataformas en línea y la aparición de redes sociales que crean más noticias e información de las que cualquier persona puede consumir "el enfoque tradicional de fijación de la agenda que traza una lista de asuntos o atributos tal y como aparece en la cobertura de noticias y luego en encuestas públicas parece insuficiente para captar la complejidad del entorno mediático actual" (Guo y Vargo, 2015, p. $\left.559^{10}\right)$. Neuman et ál. (2014) coinciden al afirmar que: "en un mundo de medios digitales en evolución y públicos en línea, las dinámicas de las agendas de asuntos se están volviendo más complejas" (p.194 $\left.{ }^{11}\right)$.

Precisamente, con los cambios que la era de Internet trajo al ecosistema mediático, algunos investigadores ya se habían cuestionado sobre la vigencia de la teoría de agenda setting. Mientras Searles y Smith (2016) afirman que:

9 The NAS model contributes to the discussion of new media and communication theories by enabling a more nuanced representation of information networks and by offering an approach to better mea- sure the flow of such networks between different entities.

10 The traditional agenda-setting approach of tracing a list of discrete objects or attributes as it appears in news coverage and then on public polls seems insufficient to capture the complexity of the current media environment.

11 In a world of evolving digital media and online publics, the dynamics of issue agendas are becoming more complex. 
"recientes trabajos sobre la elección de los medios cuestionan la influencia continua de los medios de comunicación tradicionales en la agenda pública" (p. 2074²). Tran (2014) señala que algunos investigadores "predijeron que las características descentralizadas de la nueva comunicación mediática harían menos posible que los medios de comunicación pudieran decir a la gente en qué pensar" (p.20513). McCombs (2005), en un repaso que hace sobre el pasado, presente $y$ futuro de la investigación en agenda setting explica:

Hay muchas agendas en la sociedad contemporánea y muchas más de estas están ahora disponibles para un gran segmento del público. Por lo tanto, algunos observadores sociales predicen el final de la agenda setting al tiempo que las audiencias se fragmentan $y$ virtualmente todo el mundo tiene una única agenda de medios externos que es un compuesto altamente individualizado construido a partir de esta vasta riqueza de noticias e información en línea (p.544 $\left.{ }^{14}\right)$.

En esta línea, Neuman et ál. (2014) se preguntan quién fija la agenda en la era digital $y$ de inmediato afirman que: "proponer que el poder de la agenda pública ha pasado de las élites de los medios de comunicación y las instituciones tradicionales a la ciudadanía sería ingenuo" (p.1945); sin embargo, sí sostienen

12 Recent work on media choice calls into question the continued influence of traditional news media on the public agenda.

13 They predicted that the decentralized characteristics of the new media communication would make it less possible for the media to tell people what to think about.

14 There are many agendas in contemporary society and many more of these are now readily available to a large segment of the public. Consequently, some social observers predict the end of agenda setting as audiences fragment and virtually everyone has a unique external media agenda that is a highly individualized composite constructed from this vast wealth of online news and information.

15 To posit that the power of the public agenda has swung from media elites and establishment institutions to the citizenry would be naive. que los postulados fundacionales de la teoría de la agenda setting se verían beneficiados si se repiensan a la luz del nuevo contexto. De esta manera, agenda setting en línea (online agenda setting) se ha convertido en "una nueva frontera para los estudiosos de los medios que buscan examinar la relevancia de la teoría en el mundo de la Web 2.0" (Tran, 2014, p.205 ${ }^{16}$ ).

Tran (2014), quien en su artículo hace un exhaustivo repaso de la literatura sobre agenda setting en línea, explica que conforme la comunicación masiva en la web "se convirtió en un fenómeno omnipresente, los académicos han trasladado la investigación más allá del contexto tradicional de los medios de comunicación y han asumido la tarea de probar la hipótesis de establecimiento de la agenda con nuevas plataformas de medios" (p.205 $5^{17}$ ).

En la revisión de literatura sobre agenda setting en línea, Tran (2014) encontró 26 publicaciones en revistas académicas, las primeras del año 2002. Si bien, en los diversos estudios que han seguido la tradición de la agenda setting desde el año 1972, se ha investigado sobre el poder de fijación de la agenda de los medios, del público y de la clase política (policy agenda setting); la mayoría (casi el 70\%) de los 26 estudios revisados por Tran exploran el poder de fijación de la agenda entre los mismos medios ("intermedia agenda setting").

Las tecnologías digitales no solo han permitido a los actores políticos comunicarse directamente con la ciudadanía, también han permitido a las personas "tomar los medios entre sus manos" y publicar su propia cobertura (Sayre et ál., 2010, p.7). Para Tran (2014), el contenido generado por el usuario puede rellenar lo que queda excluido por los medios de comunicación profesionales e incluso servir de catalizador para la cobertura de los medios de comunicación, es decir, "alternativas a los principales medios de comunicación se han

\footnotetext{
16 A new frontier as media scholars seek to examine the relevance of the theory in the Web 2.0 world.

17 Became a ubiquitous phenomenon, scholars have moved research beyond the traditional media context and taken up the task of testing the agendasetting hypothesis with newer media platforms.
} 
convertido en nuevos actores en el proceso de fijación de la agenda" (Tran, 2014, p.211 ${ }^{18}$ ). En esta línea de investigación, Tran (2014) cita a Sayre et ál. (2010); Roberts, Wanta y Dzwo (2002); Siu (2008) y Lee (2005).

No obstante, hay muchos más estudios que vale la pena destacar, sobre todo los que investigan a las populares redes sociales Facebook y Twitter, por ejemplo, Groshek y Groshek (2013); Neuman et ál. (2014); Rubio (2014); Martin y Grüb (2016); Antón y Alonso (2015), así como, los que acompañan este artículo en el presente número de la Revista de Ciencias Sociales, preparados por Siles, Campos, Segura y Céspedes; Tristan y Álvarez.

En los estudios tradicionales, la agenda del público se conoce a través de encuestas de opinión pública y la pregunta sobre el problema más importante. Tal y como apuntan McCombs et ál. (2014), se trata de una muestra de la conversación sobre asuntos públicos; no obstante, hoy, gracias a las redes sociales (como Facebook y Twitter) la muestra de esta conversación es mucho más amplia $y$ diversa.

Citando a Kelly (2009), los autores reconocen que gran parte de la conversación en redes sociales gira en torno a los intereses personales de las personas $y$ tiene poco que ver con asuntos públicos, no obstante, ahora, gracias a las redes sociales, está disponible para análisis y observación una importante selección de la discusión de los medios y de la ciudadanía sobre asuntos públicos (McCombs et ál., 2014). Advierten, sin embargo, que "a pesar de su enorme tamaño, este estrato todavía es una muestra limitada de la conversación ciudadana" (p.78919).

Una de las fuentes primarias de los mensajes que conforman la conversación sobre asuntos públicos en las redes sociales son los eventos noticiosos del día (McCombs et ál., 2014). Los ciudadanos luego usan las redes

18 Alternatives to the mainstream media have become new players in the intermedia agendasetting process.

Despite its huge size, this stratum is still a limited sample of the civic conversation. sociales para comentar, distribuir o buscar información información sobre estos temas y asuntos (Meraz, 2013; Vargo et ál., 2014 citado en McCombs et ál., 2014).

Cabe preguntarse, entonces ¿de qué manera $y$ en qué medida todos estos cambios han incidido en la esfera pública y en la vida democrática?, ¿pueden tener algún impacto si pareciera que estamos ante una ciudadanía que, mayoritariamente, no se interesa por los asuntos públicos? Tal y como lo señalaba Siles et. ál., y Tristán y Alvarez en este número de la Revista.

\section{¿SON LOS CLICS SINÓNIMO DE INTERÉS Y VICEVERSA?}

En la era digital de las noticias en línea $y$ en redes sociales, todas las organizaciones noticiosas pueden monitorear minuto a minuto el comportamiento de sus audiencias. Esto era algo que en el pasado solo estaba al alcance de los noticiarios televisivos, gracias a las mediciones en tiempo real de los "ratings" de sus noticiarios y los de la competencia; sin embargo, en la actualidad, cualquier medio digital puede usar analítica (con software como Google Analytics o Chartbeat) y saber exactamente, no solo cuántos usuarios ven cada noticia sino también cuánto tiempo dedican a cada nota, entre mucha otra información que los periodistas tienen al alcance de la mano. Esto ha producido una transformación en las salas de redacción porque se ha pasado de una cultura en la que la audiencia prácticamente no se conocía y no era "realmente importante", a una en la que todo gira alrededor de la audiencia, sus "clics" y las métricas web que reportan sobre lo que hace en línea (Carazo, s.f.; Cherubini y Nielsen, 2016).

Groot y Costera (2017) exploran qué significan realmente esos clics, problematizan la relación entre estos $y$ los intereses de la audiencia. Los autores documentan algunas prácticas de los usuarios de sitios web de noticias que sí expresan su interés pero que no se traducen en clics. En un artículo previo, Costera y Groot (2015) habían identificado estas prácticas, nombrándolas y definiéndolas: 
$\diamond \quad$ chequear: de forma rápida y eficiente descubrir si algo nuevo o interesante está sucediendo, mirando los titulares más recientes;

$\diamond \quad$ monitorear: inspeccionar activamente el entorno informativo para poder entrar en acción cuando sea necesario;

$\diamond \quad$ merendar (o "snacking"): agarrar pedazos pequeños ("bits and pieces") de información de manera relajada para tener una idea de lo que está sucediendo;

$\diamond \quad$ escanear: seleccionar los aspectos más destacados de las noticias para obtener la esencia de la historia.

Es decir, los "clics solo cuentan parte de la historia" (Groot y Costera, 2017, p. 14²0). Los autores van más allá y afirman que tan importante como lo que los usuarios cliquean es aquello que no cliquean. Mencionan consideraciones pragmáticas no relacionadas con el contenido que interfieren con comportamiento de cliquear. De igual manera, afirman que los titulares pueden darle a los interesados en un tema en particular poca información como para llevarlos a cliquear la noticia o al contrario, suficiente información para no requerir de un clic. Adicionalmente, las prácticas que identificaron (chequear, monitorear, escanear, etc.) no se traducen en clics, pero sí satisfacen necesidades de información sobre asuntos públicos para los usuarios: "Nuestro argumento no es que los clics no tengan significado, solo que capturan un rango limitado de intereses o preferencias de los usuarios" (Groot y Costera, 2017, p. $14^{21}$ ).

Entonces, si al igual que lo han documentado otros estudios (Tenenboim y Cohen, 2015; Tewksbury, 2003 y Boczkowski y Mitchelstein, 2013), las investigaciones de Siles et ál. y Tristán y Álvarez (en este número), apuntan a que la población costarricense está más interesada en sucesos, fútbol y entretenimiento,

\footnotetext{
$20 \quad$ Clicks only tell part of the story

21 Our argument is not that clicks are meaningless, they just capture a limited range of users' interests or preferences.
}

no cabe duda de que se trata de las temáticas que reciben más clics pero, siguiendo a Groot y Costera (2017), cabe la posibilidad de que sin cliquear ese tipo de noticias también estén informados sobre los asuntos públicos o estén buscando estos temas en otros espacios informativos menos tradicionales.

El trabajo de la prensa en torno al caso conocido como "el Cementazo" (un escándalo de corrupción que llegó hasta las más altas esferas de los tres poderes - ejecutivo, legislativo y judicial- en Costa Rica hacia finales del año 2017) brinda índices en este sentido. En el estudio de opinión del Centro de Investigación y Estudios Políticos (сіер) de la Universidad de Costa Rica (UCR) del mes de agosto de 2017, la corrupción estaba colocada en cuarto lugar como "el principal problema del país" (mencionada por $9,2 \%$, detrás de desempleo con $18,4 \%$, inseguridad y delincuencia con $17,4 \%$ y costo de vida y situación económica con 12,9\%) (p. 20).

La prensa empezó a publicar sobre el tema de la importación de cemento en el segundo semestre de 2017; primero solo un medio (CRHoy.com), pero pronto las denuncias y la información dominaban la agenda mediática. Al preguntarle a la ciudadanía solo dos meses después, en octubre de 2017 (ciep, octubre de 2017, p. 25), sobre el principal problema, la corrupción había subido al segundo lugar, pasando de $9,2 \%$ a $15,4 \%$ (cuadro 1), una diferencia significativa; es decir, pareciera comprobarse un efecto de fijación de la agenda mediática en la agenda del público, tal y como lo propone la teoría de la agenda setting.

Esto sugeriría que existe un poder de fijación de la agenda en temas relevantes como casos de corrupción, aunque esto no necesariamente quede reflejado en los clics de las noticias más leídas. En otras palabras, existe agenda setting al mismo tiempo en que las noticias con más participación son sobre sucesos, entretenimiento y deportes (Siles et ál., 2017; Siles et ál., en este número; Tristán y Álvarez, en este número; Democracia en digital, 2017). 


\section{CUADRO 1 \\ COSTA RICA: PRINCIPAL PROBLEMA DEL PAÍS \\ AGOSTO-OCTUBRE, 2017}

\begin{tabular}{lrlr}
\hline AGOSTO 2017 & & OCTUBRE 2017 \\
\hline Desempleo & $18,4 \%$ & Desempleo & $17,2 \%$ \\
Inseguridad y delincuencia & $17,4 \%$ & Corrupción & $15,4 \%$ \\
Costo de vida y situación económica & $12,9 \%$ & Situación económica & $11,3 \%$ \\
Corrupción & $9,2 \%$ & Inseguridad & $8,7 \%$ \\
Mala gestión de gobierno & $7,6 \%$ & Pobreza y desigualdad & $8,6 \%$ \\
Problemas con servicios de salud & $5,1 \%$ & Gestión del gobierno & $7,3 \%$ \\
\hline
\end{tabular}

Fuente: $\quad$ Elaboración propia con base en estudios de opinión de CIEP/UCR, agosto y octubre 2017.

\section{¿LEE LA CIUDADANÍA SOBRE ASUNTOS DE INTERÉS PÚBLICO POR NECESIDAD DE ORIENTACIÓN?}

La ubicuidad de las noticias en la actualidad (en medios tradicionales, en Internet $y$ en redes sociales) se traduce en que hoy, más que nunca, la ciudadanía tiene más oportunidades de encontrar noticias e información política. En este contexto, Gil de Zúñiga, Weeks y ArdèvolAbreu (2017) introducen un nuevo fenómeno mediático producto del ecosistema noticioso contemporáneo: el concepto de "las noticiasme-encuentran" (news-finds-me). Los autores lo definen como:

la medida en que las personas creen que pueden mantenerse indirectamente informadas acerca de los asuntos públicos - a pesar de no seguir activamente las noticias - a través del uso general de Internet, la información recibida de pares y las conexiones dentro de las redes sociales en línea (p. $107^{22}$ ).

22 The extent to which individuals believe they can indirectly stay informed about public affairs despite not actively following the news - through general Internet use, information received from peers, and connections within online social networks.
Los resultados de su investigación indican que muchos individuos tienen la errada percepción de que pueden ser parte de una ciudadanía informada, aún sin buscar las noticias sobre asuntos públicos activamente, puesto que creen que las noticias importantes llegarán hasta ellos o "los encontrarán". Los autores no solo concluyen que las personas que creen que las noticias los encuentran, tienen menos probabilidades de usar las fuentes tradicionales, como las noticias en televisión y los periódicos, sino también que los ciudadanos que tienen esta percepción tienen menos conocimiento sobre asuntos cívicos y políticos que aquellos que no creen que las noticias los encuentren (Gil de Zúñiga et ál., 2017).

Las implicaciones de su estudio para la comprensión de cómo las personas consumen las noticias de asuntos públicos y cómo se construye una vigorosa y saludable esfera pública son importantes. El estudio de Gil de Zúñiga et ál. (2017) sugiere que "la búsqueda activa de las noticias sigue siendo fundamental para que los ciudadanos aprendan sobre política" (p.118 ${ }^{23}$ ).

Precisamente, al preguntarse por los efectos diferenciados que puede tener la teoría de la fijación de la agenda en cada individuo, los

23 Actively seeking the news continues to be critical for citizens to learn about politics. 
estudios han reconocido que la medida en que las personas participan en la búsqueda de información puede variar y que algunas personas son más susceptibles a los efectos de la fijación de la agenda que otras (Kim, 2014). En los estudios de agenda setting, el concepto de "necesidad de orientación" ha servido para explicar estas diferencias.

El concepto de necesidad de orientación parte de la premisa de que las personas tienen una curiosidad innata sobre el mundo que les rodea y se refiere a la tendencia de los individuos a buscar información sobre los asuntos públicos en los medios (McCombs y Weaver, 1973). Se trata de un concepto psicológico, que McCombs y Weaver asocian a los estudios de agenda setting por primera vez en 1973. A pesar de que su introducción en la tendencia de estudios de agenda data de más de cuarenta años, es una explicación que no ha perdido vigencia y que sigue siendo central en algunos estudios de agenda contemporáneos (Kim, 2014; Chernov, Valenzuela y McCombs, 2011; Matthes, 2006 y 2008).

El concepto establece que entre más importante sea la necesidad o el deseo de información (es decir, la necesidad de orientación), más atención le pondrán las personas a los medios. La necesidad de orientación está definida en términos de: 1) la relevancia de la información para los individuos y 2) la incertidumbre que tengan los individuos (McCombs y Weaver, 1973).

El contexto de campaña electoral con miras a elecciones para presidente en un país con larga tradición democrática como lo es Costa Rica, es uno en el que los individuos tienen una sentida necesidad de orientación, sobre todo considerando "los débiles vínculos del electorado con los partidos políticos y la alta volatilidad de las preferencias electorales" (ciep, agosto de 2017, p. 7).

Según el primer estudio de opinión pública del ciep de la Universidad de Costa Rica de cara a las elecciones presidenciales de febrero de 2018, en julio de 2017, entre las personas "que dijeron que sí votarían y que además manifestaron estar totalmente decididas a votar (547 casos, el 54\% de las personas encuestadas) el grupo más numeroso lo representa el $42 \%$ que mencionó no tener un candidato de preferencia en este momento" (cIEP, agosto de 2017, p.7). En términos de incertidumbre y relevancia mencionados anteriormente, estas personas decididas a votar, pero indecisas sobre por quién hacerlo, tienen "alta incertidumbre", por tanto, alta necesidad de orientación $y$, por ende, la información sobre la campaña electoral es o será de alta relevancia para ellas.

Dos investigaciones que analizaron el comportamiento de los electores, una en las elecciones presidenciales 2014 (Alfaro y Gómez, 2014) y otra en la campaña 2018 (ciep, febrero de 2018), confirman la idea de una ciudadanía con necesidad de orientación.

$\mathrm{Al}$ escribir el presente artículo, todavía hay pocos datos sobre la campaña electoral 2017-2018; sin embargo, algunas investigaciones que analizaron la última campaña con miras a las elecciones presidenciales 2014 confirman la idea de una ciudadanía con necesidad de orientación. Alfaro y Gómez (2014) reportan sobre un interesante estudio de seguimiento a votantes realizado por el Programa Estado de la Nación y la firma de estudios de opinión pública unimer durante la campaña electoral 2013-2014. Se trata del primer panel electoral que se realiza en el país y da cuenta de los cambios en las preferencias de un grupo de votantes durante los meses de esa campaña.

Los autores sostienen que, en la actualidad, los costarricenses tienen identidades partidarias "débiles y poco influyentes en sus decisiones electorales" (Alfaro y Gómez, 2014, p.32), contrario a lo que sucede en otros contextos adonde la ciudadanía tiene fuertes vínculos con los partidos políticos y, por tanto, su decisión de votar y por cuál candidato hacerlo es facilitada por esos vínculos. Para ellos:

la identidad partidaria facilita la toma decisiones políticas y en las sociedades en las que esas afinidades son inexistentes o débiles, los ciudadanos se ven en la obligación de dedicar un mayor esfuerzo para reunir y procesar la información, por mínima que ésta sea, o invierten más 
tiempo consultando con sus familiares, amistades o compañeros de trabajo con la finalidad de tomar una decisión (Alfaro y Gómez, 2014, p.32).

Alfaro y Gómez (2014) explican que el panel electoral, que consistió en cinco rondas de preguntas a los ciudadanos participantes, permitió medir los efectos de las fluctuaciones de las preferencias entre todos los candidatos individualmente. La figura 1 representa cada una de las personas que participaron en el panel como una línea de color. Su preferencia electoral está definida de acuerdo al color del partido que dijeron apoyar, de manera que, cuando un individuo cambió de preferencia, cambia el color de la línea que lo representa. De esa forma, es posible ver, de manera agregada, los comportamientos individuales de toda la población estudiada a lo largo de la campaña electoral.

FIGURA 1

PANEL ELECTORAL: TRAYECTORIAS INDIVIDUALES DE LA PREFERENCIA ELECTORAL CAMPAÑA PRESIDENCIAL, 2013-2014

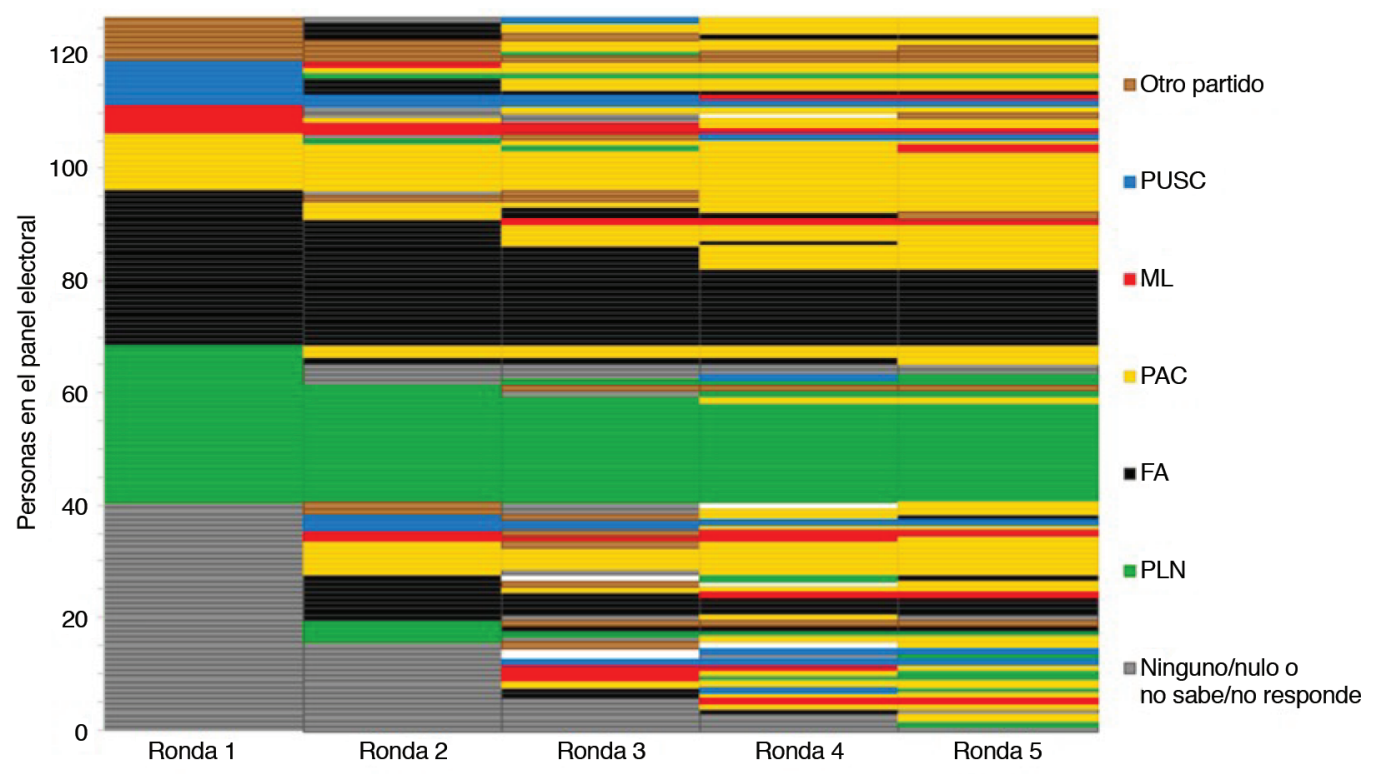

a/ Cada individuo es una línea. Cambios en el color de la línea indica un cambio en la preferencia electoral.

Fuente: $\quad$ Alfaro y Gómez, 2014, p.36.

Como parte de la investigación, se le preguntó a los participantes del panel por los tres aspectos que más pesaron en sus preferencias: "El candidato (37\%)" lideró las respuestas, pero fue seguido por "los debates (19\%, especialmente entre los decididos a votar pero sin candidato de preferencia) y el programa (16\%)" (Alfaro y Gómez, 2014, p.38), ambos aspectos que, necesariamente, requieren de seguimiento a información mediada sobre la campaña por parte de los votantes.

Durante la campaña electoral 20172018, el ciep repitió el ejercicio de panel electoral. El grupo de investigadores entrevistó a las mismas personas en cinco momentos distintos de la contienda y pudieron así "detectar los movimientos de sus comportamientos y la dirección de esos cambios" (p. 6). La 
figura 2 ilustra los movimientos documentados por el ciep $y$, al igual que el ejercicio de la campaña 2014 que se puede ver en la figura
1, los movimientos son testimonio de un escenario de "incertidumbre y volatilidad de las preferencias" (p. 6).

FIGURA 2

PANEL ELECTORAL: TRAYECTORIAS INDIVIDUALES DE LA PREFERENCIA ELECTORAL CAMPAÑA PRESIDENCIAL, 2017-2018

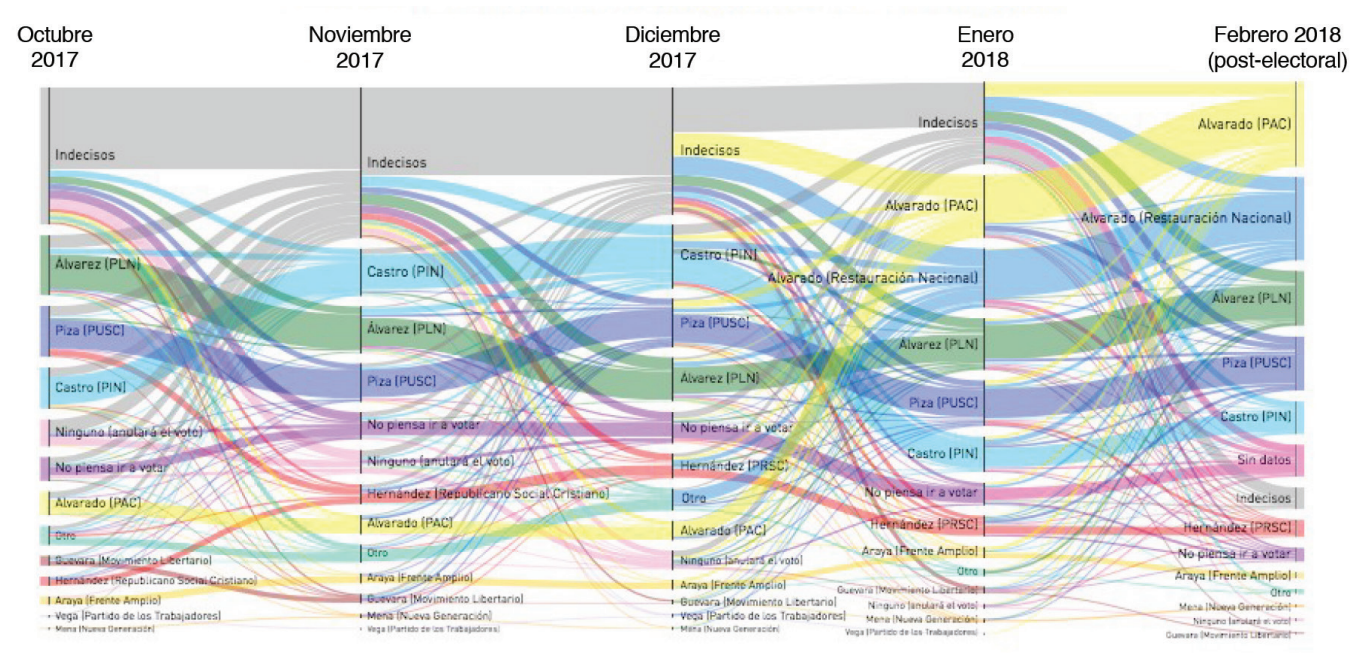

Fuente: $\quad$ CIEP, febrero de 2018.

En la campaña 2017-2018, el CIEP (enero de 2018) también preguntó sobre los factores que inciden en la decisión de voto y fueron las noticias ("mucho" 39\%, "algo" 21\%) y los debates ("mucho" 36\% y "algo" 20\%) los que más influyen (p. 14).

Sin que la "necesidad de orientación" haya sido una variable de estudio en ninguno de los dos paneles electorales (Alfaro y Gómez, 2014, ciep, 2018), los hallazgos de ambos trabajos y las respuestas de estos estudios $y$ otros sobre los factores que inciden en la decisión de voto, dan importantes pistas sobre el comportamiento de los votantes, especialmente los indecisos y por tanto, sobre el comportamiento de los consumidores de información noticiosa durante una campaña electoral.

Por otro lado, si bien los artículos de Siles et ál. y Tristán y Álvarez (en este número) apuntan a que no hay preferencia por asuntos públicos en los meses en que ambos grupos de investigadores realizaron su estudio, dos análisis de los meses de enero 2014 y enero 2018 previo a las elecciones del primer domingo de febrero en ambos años, parecen indicar que, como se ha demostrado en otros países (Tewksbury, 2003 y 2006; Boczkowski, Mitchelstein y Walter, 2012) y tal como apuntan algunos de los resultados de los paneles electorales de 20132014 y 2017-2018 (Alfaro y Gómez, 2014 y cIEP, febrero de 2018), en Costa Rica también es una tendencia que se revierte en tiempos de campaña electoral.

Un análisis de Carazo (2017) de las 100 publicaciones (posts) con más participación (engagement) en los perfiles de Facebook de dos de los principales medios del país, $\mathrm{La} \mathrm{Na-}$ ción y CRHoy.com durante enero de 2014, arroja que en ambos medios, mayoritariamente, los posts con más participación eran sobre la 
campaña electoral $(71 \%$ en el perfil de Facebook de La Nación y 52\% en el perfil de CRHoy. com, ver figura 3). Más contundentes son los resultados al comprobar que de los 100 posts con mayor participación en cada uno de los dos perfiles, en ambos, sobresalieron las publicaciones sobre asuntos públicos (90\% en La Nación y $71 \%$ en CRHoy.com).

FIGURA 3

100 POSTS CON MÁS PARTICIPACIÓN

FACEBOOK DE LA NACIÓN Y DE CRHOY.COM

ENERO, 2014

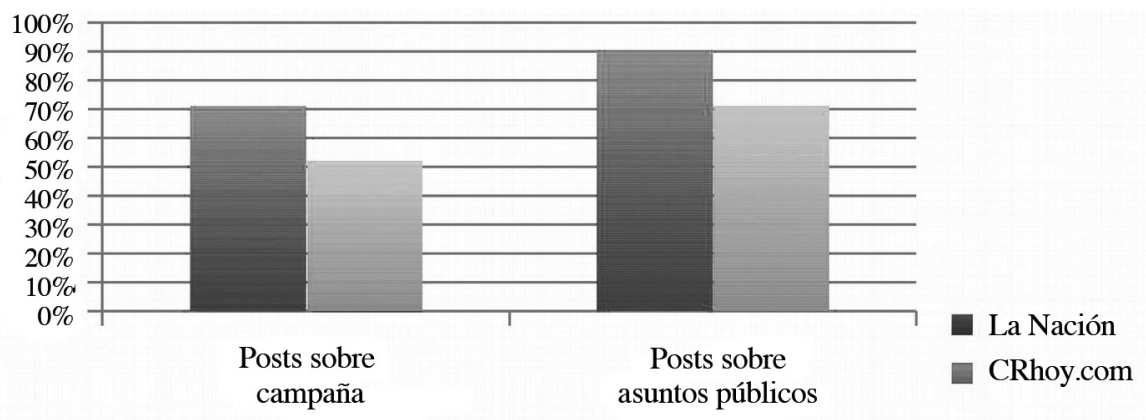

Fuente: Elaboración propia con base en estudio Carazo (2017).

En la campaña 2017-2018, un estudio en curso del Centro de Investigación en Comunicación (сісом) de la Universidad de Costa Rica (ucR), el 10\% de los posts con más participación ("engagement") publicados en los perfiles en Facebook de La Nación y CRHoy. com, demuestra que el interés por asuntos públicos es mucho menor (entre 30\% y 40\%) en comparación con la campaña 2013-2014; sin embargo, resulta interesante el marcado incremento en el interés por asuntos públicos en el mes de enero (2018) en $\mathrm{La} \mathrm{Na}$ ción y en diciembre (2017) y enero (2018) en CRHoy.com (ver Figura 4). 
FIGURA 4

INTERÉS SOBRE ASUNTOS PÚBLICOS EN POSTS CON MÁS PARTICIPACIÓN

FACEBOOK DE LA NACIÓN Y CRHOY.COM

AGOSTO DE 2017, DICIEMBRE DE 2017

Y ENERO DE 2018

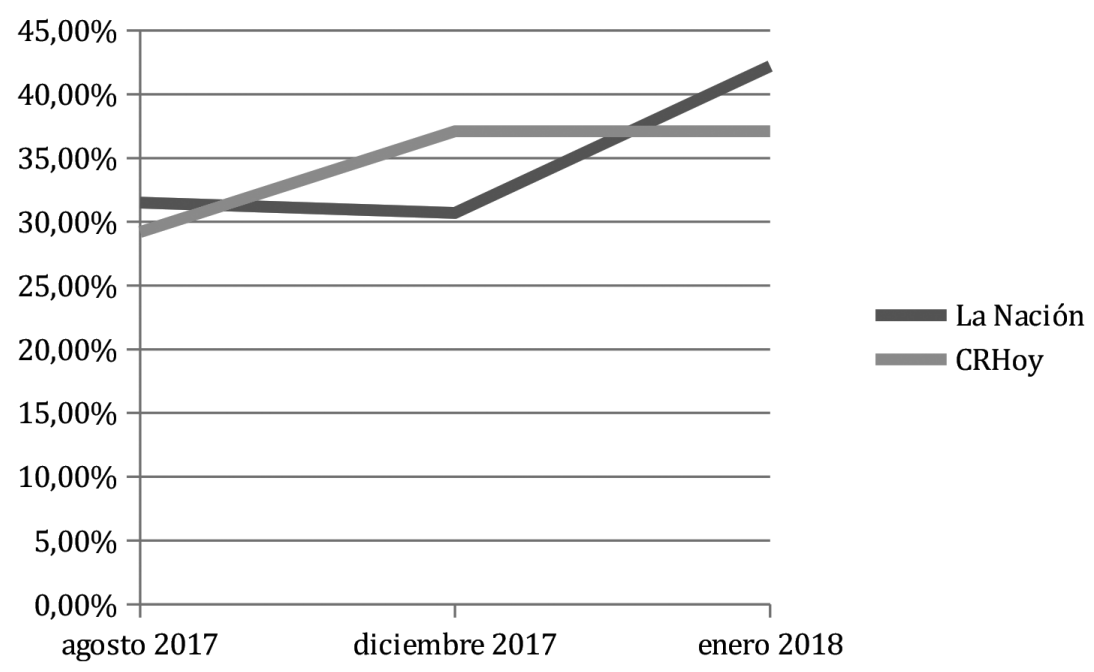

Fuente: Elaboración propia con base en datos de estudio en curso del cICom/ucR, agosto de 2017, diciembre de 2017 y enero de 2018.

Cuando se comparan estos datos con los que arrojan los estudios de Siles et ál. y Tristán y Álvarez (en este número) para periodos no electorales, pareciera comprobarse lo que señalan Boczkowski et ál. (2012) de que "el público está más atento a las noticias políticas durante las etapas finales de las campañas electorales" (p.35024). Esto se corroborará en estudios que se están realizando (al cierre de este número) durante la campaña electoral 2017-2018.

\section{¿EL INTERÉS POR FARÁNDULA Y DEPORTES SIGNIFICA QUE NO SE PARTICIPA EN POLÍTICA?}

Cada vez más, las personas usan las redes sociales, entre otras cosas, para informarse y para participar. Según el portal de estadísticas y estudios statista.com, en el año 2017, Facebook tenía más de dos mil millones de usuarios activos. En Estados Unidos, en

24 The public is more attentive to political news during the final stages of electoral campaigns. agosto de 2016 (durante la campaña electoral con miras a las elecciones presidenciales de noviembre de 2016), el $62 \%$ de los estadounidenses reportó recibir las noticias a través de las redes sociales (Shearer y Gottfried, septiembre de 2017). En Costa Rica, el consumo de noticias en redes sociales viene en aumento desde hace varios años. En el año 2015, un 40\% de la población reportaba el consumo de noticias como una de las cuatro actividades principales realizadas en Facebook (El Financiero/unimer, 2015). En octubre de 2017, las redes sociales eran "el principal medio para informarse" de $23,56 \%$ de la población en general y del 39,3\% de los menores de 34 años (CIEP/UcR, octubre de 2017, p. 26).

Estos datos son relevantes porque un pilar fundamental de la vida en democracia es que la ciudadanía se mantenga informada sobre asuntos públicos. Esto se logra a través del acceso y la exposición a información sobre asuntos públicos y "las redes sociales, como un lugar en el que las personas comparten 
contenido entre sí, permiten un nuevo medio de transmisión de información política" (Bode, 2016 , p. $1^{25}$ ). Lo interesante es que no solo se hace referencia a la transmisión de información política, sino también a la "expresión y participación política".

Para Bode et ál. (2014) "Internet puede estar extendiendo la influencia de la expresión política en formas novedosas y duraderas" (p. 417) porque, al fin y al cabo, la expresión política, aunque importante por sí misma, "también se considera un componente clave de la participación política" (p. 417). Ya lo decía Castells (2009), como mínimo, la comunicación digital facilita las interacciones de muchos-a-muchos, de uno-a-muchos y de muchos-a-uno, las cuales combinadas permiten que la participación alcance niveles nunca antes vistos.

Bode (2016), citando a Gil de Zuñiga et ál. (2010), lo explica así: "las vías de comunicación para la participación se expanden continuamente para incluir nuevos tipos de comunicación facilitados por la tecnología, y los sitios de redes sociales proporcionan otra vía potencial" (pp. 417-41826). Y es que la facilidad de comunicación que la Internet permite ha reducido el costo de la participación en línea (Gil de Zúñiga et ál., 2010; Margetts et ál., 2016).

Tradicionalmente, la participación política tenía cuatro dimensiones: votación, actividad de campaña, contactos con los funcionarios y actividades colectivas (Gil de Zúñiga et ál, 2010, p. 38). Hoy, en la sociedad digital donde reinan las redes sociales, la participación política ha tomado nuevas formas:

Las redes sociales en Internet han cambiado el contexto en el que los ciudadanos operan e influyen en sus decisiones sobre si participar políticamente. Los cambios en el entorno de la información

25 Social media, as a venue in which people share content with one another, allows for a new means of transmission of political information.

The communication pathways to participation are continually expanding to include new types of communication facilitated by technology, and social networking sites provide another potential pathway. afectan la forma en que los ciudadanos buscan $y$ encuentran información política, afectan la naturaleza de la información que reciben y reducen los costos de interacción entre ellos; cambian la escala $y$ la forma de las redes sociales $y$ de información de las personas y sus posiciones dentro de ellas, lo que repercute en los costos de la decisión de acción colectiva (Margetts et ál., 2016, p. 1127).

Para estos autores, cualquier forma de interacción en redes sociales (firmar una petición, compartir un contenido, darle "me gusta" a un perfil de un candidato...) es un "pequeño acto de participación política" (Margetts et ál., 2016, p.31). En la encuesta postelectoral que el ciep (febrero de 2014) realizó después de las elecciones presidenciales, se documentó que, además de ejercer el derecho al voto, la forma como más personas dijeron haber participado en la campaña electoral fue a través de redes sociales (figura 5).

$27 \quad$ Internet-based social media reshape the context within which citizens operate and influence their decisions about whether to participate politically. Changes to the information environment affect the way that citizens seed and find political information, affect the nature of the information they receive, and reduce the costs of interacting with each other; they change the scale and shape of individuals'social and information networks and their positions within them, impacting upon the costs in the collective action decision. 


FIGURA 5
COSTA RICA: PARTICIPACIÓN EN EL PROCESO ELECTORAL
FEBRERO, 2014

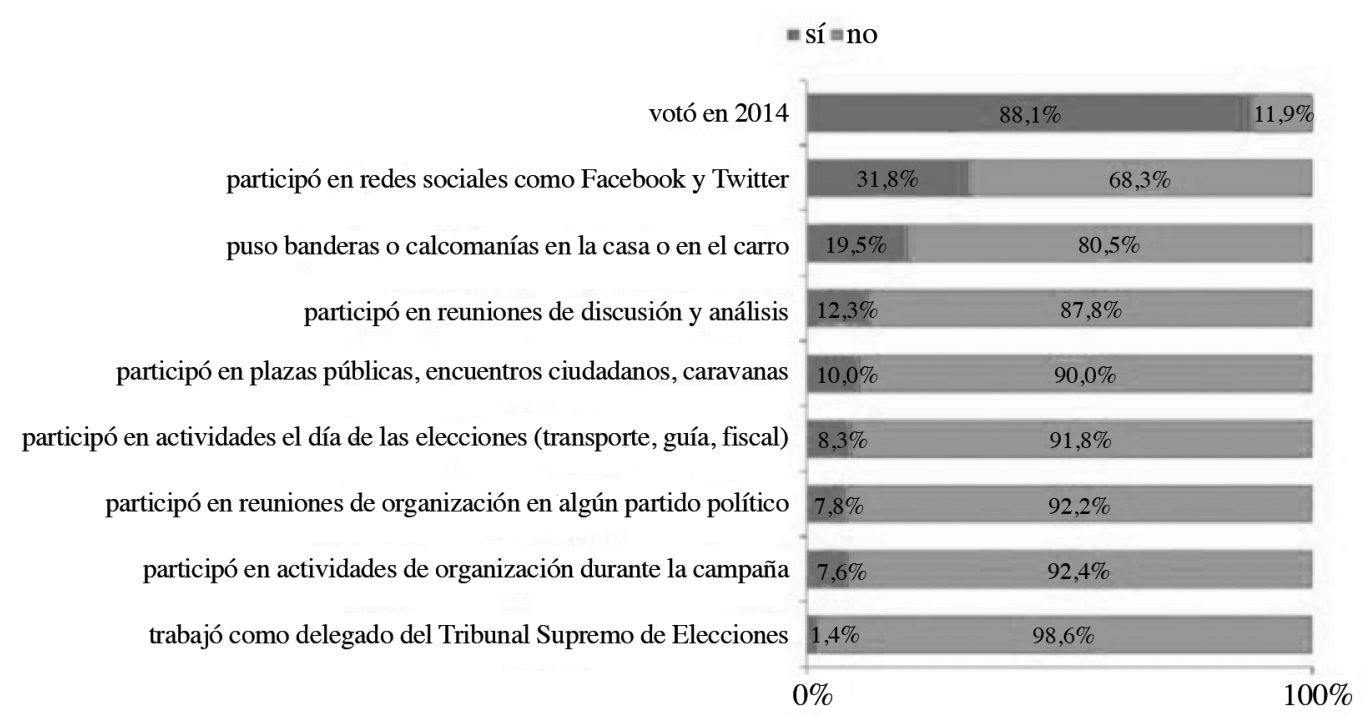

Fuente: Estudio de opinión pública post-electoral, CIEP/UcR, febrero de 2014 .

Otro ejemplo es el que presenta Kotras (2018) en su artículo en este número: frente a los acontecimientos de la polémica elección del directorio del congreso costarricense el 1 de mayo de 2017, solo en uno de los perfiles en Facebook de los múltiples medios noticiosos del país, en cuatro días (del 30 de abril al 3 de mayo) se publicaron 51 noticias relacionadas con el tema, las cuales generaron 10299 comentarios.

Si bien se trata de parte del mismo mes en el que Tristán y Álvarez (en este número) encuentran que las personas prefieren notas sobre asuntos que no son de interés público, principalmente deportes $y$ entretenimiento, no se puede obviar lo que bien apunta Kotras (en este número): "estos miles de comentarios son interesantes pues constituyen un momento importante de expresión política por parte de los usuarios de las redes sociales: se produjeron opiniones, pronósticos, críticas, muestras de apoyo o análisis de la situación política nacional en grandes cantidades en esta ocasión".
Así, reducir la participación política en redes sociales a la lectura de noticias sobre asuntos públicos podría ser engañoso. No hay todavía evidencia para concluir que la "brecha de las noticias" y los "pequeños actos de participación política" no puedan coexistir o que su coexistencia sea necesariamente perjudicial para la esfera pública. Asimismo, sería pertinente interrogar con mayor detalle los actos de participación política que suelen agruparse en el nombre del engagement para comprender mejor su significado para los públicos contemporáneos.

\section{SE ABREN INTERROGANTES PARA ESTUDIAR NUEVAS Y COMPLEJAS DINÁMICAS}

Los cambios que han provocado las redes sociales en las prácticas ciudadanas por un lado, para mantenerse informado y por otro, para expresar y compartir opiniones sobre asuntos públicos, así como participar en política, son nada menos que excepcionales. 
En este artículo se discutió, primero, lo que el advenimiento del ecosistema digital ha significado para el paradigma de medios de comunicación con poder para fijar la agenda de debate $y$ discusión en la esfera pública. Hasta hace relativamente poco tiempo, todavía a inicios del siglo xxi, cientos de estudios de la trayectoria de la teoría de agenda setting confirmaban - con distintos matices, cierto, pero con contundencia- que los medios de comunicación (tradicionales) estaban en un lugar de privilegio para fijar la agenda de discusión pública. Sin embargo, el vertiginoso crecimiento de la oferta de medios digitales y de las redes sociales complejizó las dinámicas entre las agendas mediática, política y ciudadana de manera tal que las respuestas a las preguntas sobre quién fija la agenda de discusión pública y cómo se fija dicha agenda ya no son tan categóricas. Es más, están siendo repensandas y reevaluadas por estudiosos de todo el mundo.

Interesa detenerse en el cuestionamiento de la teoría de la agenda setting en relación con el contexto mediático hipersaturado: pareciera que los postulados y la propuesta metodológica tradicional de la teoría son insuficientes para explicar un entorno tan complejo y con capacidades casi infinitas de individualización de la oferta noticiosa ¿Continua siendo válido hablar de "una" agenda mediática cuando los mismos medios tienen múltiples agendas que difieren, por lo menos, en cada una de las diversas plataformas en las que ofrecen su contenido noticioso?, ¿cómo hablar de "una" agenda de los medios cuando cada persona usuaria consume un "mix" de noticias a la medida?

Por otro lado, el método tradicional de conocer la agenda del público a través de la pregunta sobre el principal problema del país en encuestas de opinión pública resulta, como mínimo, anacrónico. Nuevas herramientas y nuevos métodos como los utilizados por Siles et ál. (en este número), que sustituyen los "autoinformes" por el análisis de big data y de la llamada "huella digital" que dejan las audiencias, contribuyen a dibujar un panorama más preciso y más completo del complejo ecosistema mediático y los comportamientos y prácticas de la ciudadanía.
Otros cuestionamientos que obligan a repensar la vigencia de la teoría de la agenda setting en el mundo en el que la comunicación digital es omnipresente se relacionan con las posibilidades que tiene la clase política de prescindir de los medios para comunicarse con la ciudadanía. El paradigma clásico de los medios como únicos intermediarios está en vía de extinción si no es que ya quedó en el pasado ¿Cómo cambia, entonces, la construcción de la esfera pública en un contexto en que los medios de comunicación no son ya los intermediarios obligados entre la clase política y la ciudadanía? No es un asunto menor la multiplicación de las posibilidades que los mismos ciudadanos tienen hoy para comunicarse, para informar e informarse, para participar y para incidir. Esto también obliga a una reevaluación de la teoría de la agenda.

Precisamente, la segunda parte de este artículo se detiene a reflexionar sobre todo lo anterior desde una perspectiva ciudadana, presentando algunas consideraciones $y$ ejemplos en torno a cuestiones que podrían contribuir a relativizar la preferencia que los usuarios demuestran por las noticias no relacionadas con asuntos de interés público como deportes, sucesos y entretenimiento.

En primer lugar, se discutió sobre la problematización de lo que significa un clic. El mundo de las noticias digitales (en medios en línea $y$ en redes sociales) está regido por los clics. Esto no solo ha redefinido la relación de los medios y las audiencias sino que ha traído importantes cambios a la industria periodística con la incorporación de análisis de métricas web en el día a día de las salas de redacción (Carazo, s.f; Cherubini y Nielsen, 2016). No obstante, como bien lo señalan Groot y Costera (2017), los clics no cuentan toda la historia y todavía debe hacerse mucha investigación, sobre todo cualitativa, para completar el rompecabezas $y$ tener una mejor comprensión de qué significa y qué no significa un clic.

El ejemplo que se presenta en este artículo sobre el ascenso de la percepción de la corrupción como principal problema del país en momentos en que la agenda mediática está dominada por la cobertura del caso de la 
importación de cemento chino, al mismo tiempo que siguen siendo las noticias "light" las que presentan mayor interacción (engagement) en redes sociales, deja más preguntas que respuestas y exige que se profundice la investigación y que se acuda a una mayor diversidad de métodos, incluyendo cualitativos.

En segundo lugar, se discutió en torno a la necesidad de orientación como generadora de interés por asuntos públicos. En los artículos de Siles et ál. y de Tristán y Álvarez (en este número) queda documentada la preferencia de los usuarios por las noticias sobre asuntos que no son de este tipo. Sin embargo, en este artículo se plantea que, tal y como se ha comprobado para otros contextos, pareciera que en Costa Rica esta tendencia tiende a revertirse en tiempos de campaña electoral cuando la ciudadanía necesita orientación y sigue las publicaciones de los medios de comunicación para informarse sobre los candidatos y sus propuestas, con el fin de tomar una decisión informada para votar. Se trata de un fenómeno que no ha sido objeto de estudio per se en nuestro país y que se está investigando por primera vez en la campaña electoral 2017-2018: no obstante, este artículo presenta algunas pistas que apuntan a que, efectivamente, la tendencia se revierte $y$ en época de campaña electoral, por lo menos durante la etapa final, el público sí pone más atención a los temas de interés público relacionados con la contienda.

Ahora bien, la pregunta que viene a la mente es si esto es necesariamente bueno ¿Qué nos dice de una ciudadanía que pareciera solo mostrar preferencia por asuntos de interés público cada cuatro años? Lo ideal, sería, por supuesto, que el consumo de noticias sobre asuntos de interés público se evidenciara de manera más sostenida y no solo en ciertos periodos "especiales".

Esto lleva al tercer argumento relacionado con la discusión sobre las formas en que los ciudadanos participan en política en la actualidad. La nueva era digital ha potenciado no solo el acceso a la información política, que ya de por sí es importante como un primer paso hacia la participación, sino que también se han multiplicado las posibilidades, las narrativas $y$ los formatos de expresión política, que es también, un componente clave de la participación política (Bode et ál., 2014). Es indiscutible que se han trascendido las tradicionales formas de participación (votación, actividad de campaña, contactos con los funcionarios y actividades colectivas) y actualmente los ciudadanos y las ciudadanas, "prosumidores", participan no solo produciendo videos, memes y otros tipos de contenido, sino también compartiendo, comentando $y$ reaccionando en redes sociales. Todas estas formas de interacción en redes son, en palabras de Margetts et ál. (2016) "pequeños actos de participación política".

Esto es reconocido por la ciudadanía, puesto que al preguntárseles cómo participaron en el proceso electoral del año 2014, la respuesta mayoritaria fue (además de votar, por supuesto), "participar en redes sociales" (CIEp/ucr, febrero de 2014). No obstante, a pesar de este reconocimiento, si algo queda claro es que deben interrogarse con mayor detalle estas nuevas maneras de participación para comprender qué significan para las personas ciudadanas.

Entonces, es cierto que hay evidencia de una "brecha de las noticias" pero también la hay de una ciudadanía, por lo menos, con algún grado de interés por asuntos públicos y que participa en política en el mundo digital. Es un hecho que ambos fenómenos coexisten. La pregunta es ¿qué significa esto para la esfera pública y para la construcción de la vida en democracia?, ¿anula un fenómeno al otro?, ¿hay periodos en que uno de ellos sobresale?, ¿cuáles son los periodos en que hay más interés por asuntos públicos y por qué?, y sobre todo, ¿qué acciones se pueden o se deben tomar para fortalecer un sano y vigoroso debate cívico, plural y democrático?

\section{REFERENCIAS}

Alfaro, R. y Gómez, S. (2014). Análisis electoral y de partidos políticos en Costa Rica. Ponencia preparada para el Informe Estado de la Nación. San José: PEN. Recuperado de http://estadonacion. or.cr/files/biblioteca_virtual/020/politica/ Alfaro\&Gomez2014.pdf 
Antón Crespo, M., y Alonso del Barrio, E. (2015). El 'trending topic' frente a la 'agenda setting'. Estudios Sobre El Mensaje Periodístico.Núm. Especial Diciembre, 23-34.

Bell, E. (2016). Facebook is eating the world. Columbia Journalism Review. Recuperado de http://www.cjr.org/analysis/facebook_and_media.php

Boczkowski, P. , Mitchelstein, E., y Walter, M. (2012). When Burglar Alarms Sound, Do Monitorial Citizens Pay Attention to Them? The Online News Choices of Journalists and Consumers During and After the 2008 U.S. Election Cycle. Political Communication, 29 (4), 347366. doi:10.1080/10584609.2012.722173

Boczkowski, P.J. y Mitchelstein, E. (2013). The News Gap: When the Information Preferences of the Media and the Public Diverge. Cambridge, ма: міт Press.

Bode, L. (2012). Facebooking it to the polls: A study in online social networking and political behavior. Journal of Information Technology \& Politics, 9, 352-369. doi: $10.1080=19331681.2012 .709045$

Bode, L. (2016). Political news in the news feed: Learning politics from social media. Mass Communication and Society, 19(1), 24-48.

Bode, L., Vraga, E., Borah, P., y Shah, D. (2014). A new space for political behavior: Political social networking and its democratic consequences. Journal of Computer-Mediated Communication, 19, 414-429. doi: 10.1111=jcc4.12048

Carazo, C. (junio de 2017). La agenda de los medios en periodo de campaña electoral. Ponencia presentada en Primer Foro Institucional 2017: Libertad de Expresión, Comunicación y Democracia. Universidad de Costa Rica.

Carazo, C. (s.f.) (en prensa). Operacionalización de la analítica web y la optimización en periodismo. El caso de los medios del Grupo Nación en Costa Rica. En Siles, I. (Ed.). Comunicación y tecnología. San José, Costa Rica: Editorial de la Universidad de Costa Rica.
Carroll, C. y McCombs, M. (2003). Agendasetting Effects of Business News on the Public's Images and Opinions about Major Corporations. Corporate Reputation Review 6 (1), 36-46. doi:10.1057/palgrave.crr.1540188.

Castells, M. (2009). Comunicación y poder. Barcelona, España: Alianza.

Chernov, G., Valenzuela, S., y McCombs, M. (2011). An experimental comparison of two perspectives on the concept of need for orientation in agenda setting theory. Journalism \& Mass Communication Quarterly, 88, 142-155.

Cherubini, F. y Nielsen, R. (2016). Editorial Analytics: How News Media Are Developing and Using Audience Data and Metrics. Digital News Project 2016. Oxford: Reuters Institute for the Study of Journalism. Recuperado de https:// reutersinstitute.politics.ox.ac.uk/sites/ default/ files/Editorial\%20analytics $\% 20$ -\%20how\%20news\%20media\%20are\%20 developing\%20and\%20using\%20audience\%20data\%20and\%20metrics.pdf

Ciep/ucr (febrero de 2014). Informe de la encuesta post-electoral Febrero 2014. Recuperado de https://ciep.ucr.ac.cr/images/INFORMESUOP/Informe-encuestaCIEP-post-electoral-febrero-2014.pdf

CIEP/UCR (agosto de 2017). Informe de Resultados de la Encuesta de Opinión Sociopolítica realizada en julio de 2017. Recuperado de https://www.ciep.ucr.ac.cr/images/ INFORMESUOP/Informe-encuestajulio-2017.pdf

CIEP/UCR (octubre de 2017). Informe de Resultados de la Encuesta de Opinión Sociopolítica realizada en octubre de 2017. Recuperado de https:/www.ciep.ucr.ac.cr/images/ INFORMESUOP/Informe-encuestaoctubre-2017.pdf

Costera, I. y Groot, T. (2015). Checking, sharing, clicking and linking: Changing patterns of news use between 2004 and 2014. Digital Journalism 3(5), 664-679.

Democracia en digital (2017). Recuperado de http://www.democraciaendigital.com/

El Financiero/unimer (2015). Red 506. San José: El Financiero. 
Gil de Zúñiga, H., Veenstra, A. Vraga, E. y Shah, D. V. (2010). Digital democracy: Re-imagining pathways to political participation. Journal of Information Technology and Politics 7(1), 36-51.

Gil de Zúñiga, H., Weeks, B. y Ardèvol-Abreu, A. (2017). Effects of the News-Finds-Me Perception in Communication: Social Media Use Implications for News Seeking and Learning About Politics. Journal of Computer-Mediated Communication, 22, 105-123. doi:10.1111/jcc4.12185

Groot, T., y Costera, I. (2017). What clicks actually mean: Exploring digital news user practices. Journalism, I-16. Recuperado de http://journals.sagepub. com/doi/10.1177/1464884916688290\#arti cleCitationDownloadContainer

Groshek, J., y Groshek, M. C. (2013). Agenda trending: Reciprocity and the predictive capacity of social networking sites in intermedia agenda setting across topics over time. Media and Communication, 1(1), 15.

Guo, L. (2012). The application of social network analysis in agenda setting research: A methodological exploration. Journal of Broadcasting \& Electronic Media, 56(4), 616-631.

Guo, L., $y$ Vargo, C. (2015). The power of message networks: A big-data analysis of the network agenda setting model and issueownership. Mass Communication and Society, 18(5), 557-576.

Kelly, R. (2009). Twitter Study. San Antonio, тx: Pear Analytics. En McCombs, M. E., Shaw, D. L., $y$ Weaver, D. H. (2014). New directions in agenda-setting theory and research. Mass Communication and Society, 17(6), 781-802.

Kim, Y. (2014). Contingent factors of agenda setting effects: How need for orientation, issue obtrusiveness, and message tone influence issue salience and attitude strength. En Johnson, T. (ed). Agenda Setting in a 2.0 World. New Agendas in Communication. 65-81.

Kiousis, S., Mitrook, M., Wu, X. y Seltzer, T. (2006). First-and Second-level Agenda-
Building and Agenda-Setting Effects: Exploring the Linkages Among Candidate News Releases, Media Coverage, and Public Opinion During the 2002 Florida Gubernatorial Election. Journal of Public Relations Research 18 (3), 265-285. doi:10.1207/s1532754xjprr 1803_4.

Kotras, B. (2018). Materialidad, formas y contenidos del espacio público en línea. La elección de Gonzalo Ramírez vista por los usuarios de Facebook (en este número).

Lee, B., Lancendorfer, K. M., y Lee, K. J. (2005). Agenda-setting and the Internet: The intermedia influence of Internet bulletin boards on newspaper coverage of the 2000 general election in South Korea. Asian Journal of Communication, 15(1), 57-71.

Margetts, H., John, P., Hale, S., y Yasseri, T. (2015). Political turbulence: How social media shape collective action. Princeton University Press.

Martin, S., y Grüb, B. (2016). Towards a process of agenda setting driven by social media. International Journal of Energy Sector Management, 10(1), 38-55.

Matthes, J. (2006). The Need For Orientation Towards News Media: Revising And Validating A Classic Concept. International Journal Of Public Opinion Research, 18(4), 422-444. doi:10.1093/ ijpo/edh118

Matthes, J. (2008). Need For Orientation As A Predictor Of Agenda-Setting Effects: Causal Evidence From A Two-Wave Panel Study. International Journal Of Public Opinion Research, 20(4), 440-453.

McCombs, M. (2005). A Look at Agendasetting: past, present and future. Journalism Studies, 6(4), 543-557. doi: 10.1080/14616700500250438

McCombs, M. (2014). Setting the agenda: Mass media and public opinion. (2 ed.) United Kingdom: Polity Press.

McCombs, M. E., Shaw, D. L., y Weaver, D. H. (2014). New directions in agendasetting theory and research. Mass Communication and Society, 17(6), 781-802. 
McCombs, M. $y$ Reynolds, A. (2009). How the News Shapes Our Civic Agenda. En Media Effects: Advances in Theory and Research. (3 ed.), editado por Jennings Bryant and Mary Beth Oliver, 1-16. New York, ny: Routledge.

McCombs, M.E. y Weaver, D. (1973). Voters' need for orientation and use of mass communication. Ponencia presentada en la International Communication Association. Recuperado de http://files. eric.ed.gov/fulltext/ED077061.pdf

Mitchell, A. (7 de julio de 2016). Key findings on the traits and habits of the modern news consumer. En Pew Research Center. Recuperado de http://www.pewresearch. org/fact-tank/2016/07/07/modern-newsconsumer/

Neuman, R.W., Guggenheim, L., Mo Jang, S., y Bae, S. Y. (2014). The dynamics of public attention: Agenda setting theory meets big data. Journal of Communication, 64(2), 193-214.

Roberts, M., Wanta, W., y Dzwo, T. H. (2002). Agenda setting and issue salience online. Communication research, 29(4), 452-465.

Rubio García, R. (2014). Twitter y la teoría de la Agenda-Setting: mensajes de la opinión pública digital. Estudios sobre el mensaje periodístico, 20(1), 249-265.

Sayre, B., Bode, L., Shah, D., Wilcox, D., y Shah, C. (2010). Agenda setting in a digital age: Tracking attention to California Proposition 8 in social media, online news and conventional news. Policy \& Internet, 2(2), 7-32.

Searles, K., y Smith, G. (2016). Who's the boss? setting the agenda in a fragmented media environment. International Journal of Communication, 10, 22.

Shearer, E. y Gottfried, J. (7 de septiembre de 2017). News Use Across Social Media Platforms. Pew Research Center. Recuperado de http://www.journalism. org/2017/09/07/news-use-across-socialmedia-platforms-2017/

Siles, I., Campos, P., Ramírez, D., Segura, A. y Céspedes, A. (2017). Sitios costarricenses de noticias en Facebook: ¿Qué "likean", comentan y comparten sus usuarios? Ponencia presentada en las VII Jornadas de Investigación, Acción Social y Docencia. Septiembre 19-21. Centro de Investigación en Comunicación. Universidad de Costa Rica.

Siles, I., Campos, P., Segura, A. y Céspedes, A. (2018). Sitios costarricenses de noticias en Facebook: ¿Qué "likean", comentan y comparten sus usuarios? (en este número).

Siu, W. (2008). Source Agenda Setting: Inter Media Influence In The Food Safety Scandal. Journal of Communications Research, 1(4), 355-381.

Tenenboim, O. y Cohen, A.A. (2015). What prompts users to click and comment: A longitudinal study of online news. Journalism 16(2), 198-217.

Tewksbury, D. (2003). What do Americans really want to know? Tracking the behavior of news readers on the Internet. Journal of Communication, 53, 694-710.

Tewksbury, D. (2006). Exposure to the newer media in a presidential primary campaign. Political Communication, 23, 313-332.

Tran, H. (2014). Online agenda setting: A new frontier for theory development. En Johnson, T. (ed). Agenda Setting in a 2.0 World. New Agendas in Communication. 205-229.

Tristán, L. y Álvarez, M. (2018). Oferta y demanda en la Web: Análisis de la oferta informativa $y$ los patrones de consumo de los lectores de La Nación y CRHoy.com (en este número).

Vargo, C. J., Guo, L., McCombs, M., y Shaw, D. L. (2014). Network issue agendas on Twitter during the 2012 U.S. presidential election. Journal of Communication, 64, 296-316. doi:10. 1111/jcom.12089

Vu, H. T., Guo, L., y McCombs, M. (2014). Exploring "the world outside and the pictures in our heads". A network agenda-setting study. Journalism \& Mass Communication Quarterly, 91(4), 669-686.

Wang, X. y Shoemaker, P. (2011). What Shapes Americans' Opinion of China? Country Characteristics, Public Relations 
and Mass Media. Chinese Journal of Communication 4 (1), 1-20. doi:10.1080/ 17544750.2011.544079.

Wanta, W., Golan, G., y Lee, C. (2004). Agenda Setting and International News: Media Influence on Public Perceptions of
Foreign Nations. Journalism \& Mass Communication Quarterly 81 (2), 364377. doi:10.1177/107769900408100209.

Fecha de ingreso: 24/10/2017 Fecha de aprobación: 08/11/2017 
